\title{
On the Strong CE-Property of Convex Sets
}

\author{
M. E. Shirokov* \\ Steklov Mathematical Institute, Russian Academy of Sciences \\ Received August 14, 2006; in final form, February 22, 2007
}

\begin{abstract}
We consider a class of convex bounded subsets of a separable Banach space. This class includes all convex compact sets as well as some noncompact sets important in applications. For sets in this class, we obtain a simple criterion for the strong CE-property, i.e., the property that the convex closure of any continuous bounded function is a continuous bounded function. Some results are obtained concerning the extension of functions defined at the extreme points of a set in this class to convex or concave functions defined on the entire set with preservation of closedness and continuity. Some applications of the results in quantum information theory are considered.
\end{abstract}

DOI: $10.1134 / \mathrm{S} 000143460709012 \mathrm{X}$

Key words: compact set, continuity, convex function, concave function, convex envelope, convex closure, CE-property, topological linear space, separable Banach space.

\section{INTRODUCTION}

The notion of convex closure of a function defined on a convex subset of a topological linear space is widely used in modern convex analysis and its applications [1,2]. ${ }^{1}$ The convex closure of a function $f$ can be defined as the maximal closed convex function that does not exceed $f$. There naturally arise questions as to how to represent the convex closure by a closed analytic formula, whether it coincides with the convex envelope (the maximal convex function that does not exceed $f$ ), and what the relationship between the continuity properties of $f$ and the convex closure of $f$ is. The last question is of fundamental importance for applications in quantum information theory [4], where the convex closure of a certain function serves as a characteristic of a physical system (see Sec. 6).

The convex closure and the convex envelope of an arbitrary continuous function on a convex compact set coincide [3]. However, even in $\mathbb{R}^{3}$, there exist convex compact sets and continuous functions defined on them (in particular, concave continuous functions) whose convex closure is discontinuous. A convex compact set is said to have the CE-property if the convex closure of every concave continuous function on this set is continuous $[5,6]$. In the present paper, for a wide class of convex sets, referred to as $\mu$-compact sets, we consider the following strengthened version of the CE-property: the convex closure of every bounded continuous function on a convex $\mu$-compact set is bounded and continuous. This property can naturally be referred to as the strong CE-property. The class of $\mu$-compact sets consists of subsets of separable Banach spaces. These subsets are characterized by the fact that the barycentric mapping has a certain property, which serves as a substitute of compactness in the proof of many results, including some results in Choquet's theory [7]. This class contains all convex compact sets as well as some noncompact sets important in applications, e.g., the simplex of all probability distributions with countably many outcomes and its noncommutative analog, the set of density operators on a separable Hilbert space.

We obtain a simple criterion that permits one to establish the strong CE-property for a wide class of convex compact sets in $\mathbb{R}^{n}$ as well as for the noncompact sets indicated above. We consider some corollaries of the strong CE-property related to the problem of extending continuous closed functions defined on the set of extreme points of a convex $\mu$-compact set to convex or concave functions defined on the entire set and possessing certain continuity properties.

\footnotetext{
*E-mail: msh@mi.ras.ru.

${ }^{1}$ The term "lower envelope" is also used [3].
} 
In Sec. 2, the definition of $\mu$-compact sets is given and a sufficient condition and a criterion for $\mu$-compactness are presented. Some auxiliary results about properties of $\mu$-compact sets, including a generalization of Choquet's barycentric decomposition theorem, are obtained.

Section 3 deals with a representation of the convex closure of an arbitrary closed lower-bounded function on a $\mu$-compact convex set. We establish that the convex envelope and the convex closure of an arbitrary continuous bounded function coincide; this assertion generalizes Corollary I.3.6 in [3].

In Sec. 4, we prove a theorem stating that the strong CE-property is equivalent to two other properties of a $\mu$-compact convex set. We use this theorem to establish the strong CE-property for all $\mathrm{P}$-sets (sets with certain continuity properties of the boundary [8]) in $\mathbb{R}^{n}$ as well as for the simplex of all probability distributions with countably many outcomes and for the set of density operators on a separable Hilbert space.

Section 5 contains some results on the extension, with closedness preserved, of closed lowerbounded functions defined on the set of extreme points of a $\mu$-compact convex set with the strong CEproperty to convex or concave functions defined on the entire set. In particular, we prove that each continuous bounded function defined on the set of extreme points has a continuous bounded convex extension with certain maximality properties. A criterion for the convex closure of a concave function to be continuous is obtained.

Section 6 deals with applications in quantum information theory.

In Sec. 7, we discuss possible generalizations of the main results of the paper to a wider class of convex sets.

\section{ON A CLASS OF CONVEX SETS}

In Secs. 2-6, we assume that $\mathscr{A}$ is a closed bounded subset of a separable Banach space. The following notation will be used:

$\operatorname{co}(\mathscr{A}), \sigma-\operatorname{co}(\mathscr{A})$, and $\overline{\operatorname{co}}(\mathscr{A})$ are the convex hull, the $\sigma$-convex hull, ${ }^{2}$ and the convex closure, respectively, of a set $\mathscr{A}$.

extr $\mathscr{A}$ is the set of extreme points of a convex set $\mathscr{A}$.

$C(\mathscr{A})$ is the set of continuous bounded functions on a set $\mathscr{A}$.

$P(\mathscr{A})$ and $\hat{P}(\mathscr{A})$ are, respectively, the set of convex continuous bounded functions on a convex set $\mathscr{A}$ and the set of functions representable as pointwise limits of monotone sequences of functions in $P(\mathscr{A})$.

co $f$ and $\overline{\mathrm{co}} f$ are, respectively, the convex envelope and the convex closure of a function $f$ defined on a convex set $\mathscr{A}[1,2]$.

$\mathfrak{P}_{n}=\left\{\left\{\pi_{i}\right\}_{i=1}^{n} \mid \pi_{i} \geq 0, \sum_{i=1}^{n} \pi_{i}=1\right\}$ is the simplex of all probability distributions with $n \leq+\infty$ outcomes.

The convex set of all Borel probability measures on a set $\mathscr{A}$, equipped with the weak convergence topology [9], will be denoted by $M_{1}^{+}(\mathscr{A})$. The measure in $M_{1}^{+}(\mathscr{A})$ concentrated at a point $x \in \mathscr{A}$ will be denoted by $\delta(x)$. Consider the barycentric mapping

$$
M_{1}^{+}(\mathscr{A}) \ni \mu \mapsto b(\mu)=\int_{\mathscr{A}} x \mu(d x) \in \overline{\mathrm{co}} \mathscr{A},
$$

where the integral is treated in the sense of Bochner. The convex closed subset of the set $M_{1}^{+}(\mathscr{A})$ of measures $\mu$ such that $b(\mu)=x \in \overline{\mathrm{co}} \mathscr{A}$ will be denoted by $M_{x}(\mathscr{A})$.

The mapping (1) is continuous (e.g., see [10, Sec. 2]); consequently, the image of any compact subset of $M_{1}^{+}(\mathscr{A})$ under the mapping (1) is a compact subset of $\overline{c 0} \mathscr{A}$. Let us single out a class of convex sets for which the converse assertion holds.

Definition 1. A set $\mathscr{A}$ is said to be $\mu$-compact if the preimage of every compact subset of $\overline{c o}(\mathscr{A})$ under the mapping (1) is a compact subset of $M_{1}^{+}(\mathscr{A})$.

\footnotetext{
${ }^{2}$ The set of all countable convex combinations of elements of $\mathscr{A}$.
} 
Note that every compact set $\mathscr{A}$ is $\mu$-compact, because the compactness of $\mathscr{A}$ implies that of $M_{1}^{+}(\mathscr{A})[9]$.

Proposition 1. A convex set $\mathscr{A}$ is $\mu$-compact if and only if, for any compact subset $\mathscr{K} \subseteq \mathscr{A}$ and any $\varepsilon>0$, there exists a compact subset $\mathscr{K}_{\varepsilon} \subseteq \mathscr{A}$ such that, for each $x \in \mathscr{K}$, the decomposition $x=\sum_{i=1}^{n} \lambda_{i} x_{i}$, where $\left\{x_{i}\right\}_{i=1}^{n} \subset \mathscr{A}$ and $\left\{\lambda_{i}\right\}_{i=1}^{n} \in \mathfrak{P}_{n}$, implies that $\sum_{i: x_{i} \in \mathscr{A} \backslash \mathscr{K}_{\varepsilon}} \lambda_{i}<\varepsilon$.

A convex set $\mathscr{A}$ is $\mu$-compact if there exists a family $F(\mathscr{A})$ of concave nonnegative functions on $\mathscr{A}$ with the following properties:

- The set $\{x \in \mathscr{A}: f(x) \leq c\}$ is relatively compact for any $f \in F(\mathscr{A})$ and $c>0$.

- For each compact subset $\mathscr{K} \subseteq \mathscr{A}$, there exists a function $f \in F(\mathscr{A})$ satisfying the inequality $\sup _{x \in \mathscr{K}} f(x)<+\infty$.

Proof. The first assertion in Proposition 1 follows from Prokhorov's theorem [9]. Indeed, the property indicated in this assertion means that the set of all measures in $M_{1}^{+}(\mathscr{A})$ with finite support and with barycenter in $\mathscr{K}$ is dense. By Lemma 1 below and Theorem 6.1 in [9], this is equivalent to the fact that the set of all measures in $M_{1}^{+}(\mathscr{A})$ with barycenter in $\mathscr{K}$ is dense.

The proof of the second assertion generalizes the argument in the proof of Proposition 2 in [11]. Let $\mathscr{K}$ be a compact subset of $\mathscr{A}$, and let $f \in F(\mathscr{A})$ be a function such that $\sup _{x \in \mathscr{K}} f(x)=c<+\infty$. For each $\varepsilon>0$, let $\mathscr{K}_{\varepsilon}=\{x \in \mathscr{A}: f(x) \leq c / \varepsilon\}$; this is a relatively compact subset of $\mathscr{A}$. Since $f$ is concave and nonnegative, we have

$$
c \geq f(x) \geq \sum_{i=1}^{n} \lambda_{i} f\left(x_{i}\right) \geq \sum_{i: x_{i} \in \mathscr{A} \backslash \mathscr{K}_{\varepsilon}} \lambda_{i} f\left(x_{i}\right)>\frac{c}{\varepsilon} \sum_{i: x_{i} \in \mathscr{A} \backslash \mathscr{K}_{\varepsilon}} \lambda_{i}
$$

for an arbitrary $x \in \mathscr{K}$ and a decomposition $x=\sum_{i=1}^{n} \lambda_{i} x_{i}$, whence it follows that $\sum_{i: x_{i} \in \mathscr{A} \backslash \overline{\mathscr{K}}_{\varepsilon}} \lambda_{i}<\varepsilon$.

Lemma 1. Let $\mathscr{A}$ be a convex set. Then each measure $\mu_{0} M_{1}^{+}(\mathscr{A})$ is the limit of a sequence $\left\{\mu_{n}\right\}$ of finitely supported measures in $M_{1}^{+}(\mathscr{A})$ such that $b\left(\mu_{n}\right)=b\left(\mu_{0}\right)$ for all $n$.

The proof of this lemma naturally generalizes that of Lemma 1 in [11].

The sufficient condition given in Proposition 1 can be used to show the $\mu$-compactness of the noncompact set $\mathfrak{P}_{+\infty}$ of all probability distributions with countably many outcomes. To this end, for $F\left(\mathfrak{P}_{+\infty}\right)$ one can take a family of functions of the form $\left\{p_{i}\right\}_{i=1}^{+\infty} \mapsto \sum_{i=1}^{+\infty} p_{i} h_{i}$, where $\left\{h_{i}\right\}_{i=1}^{+\infty}$ is an arbitrary increasing sequence of positive numbers tending to $+\infty$.

Many results of Choquet's theory, which was developed for compact convex sets, can readily be generalized to the case of $\mu$-compact convex sets. The forthcoming exposition heavily relies on the following generalization of Choquet's theorem [7].

Proposition 2. Let $\mathscr{A}$ be a $\mu$-compact set. Then each element of $\overline{\mathrm{co}}(\mathscr{A})$ is the barycenter of some measure in $M_{1}^{+}(\mathscr{A})$.

Proof. Let $x_{0} \in \overline{\mathrm{co}}(\mathscr{A})$. By the definition of convex closure, there exists a sequence $\left\{x_{n}\right\} \subseteq \operatorname{co}(\mathscr{A})$ converging to $x_{0}$. For each $n$, the element $x_{n}$ is the barycenter of some finitely supported measure $\mu_{n} \in M_{1}^{+}(\mathscr{A})$. Since the set $\mathscr{A}$ is $\mu$-compact, it follows that the sequence $\left\{\mu_{n}\right\}$ is relatively compact and hence has a partial limit $\mu_{0} \in M_{1}^{+}(\mathscr{A})$. The mapping (1) is continuous, and so $x_{0}$ is the barycenter of $\mu_{0}$. 
If $\mathscr{A}$ is a convex set, then the set $M_{1}^{+}(\mathscr{A})$ can be equipped with the following partial order, which is often called the Choquet order [7, 12]. One has $\mu \succ \nu$ if and only if

$$
\int_{\mathscr{A}} f(y) \mu(d y) \geq \int_{\mathscr{A}} f(y) \nu(d y) \quad \forall f \in P(\mathscr{A}) .
$$

A measure $\mu \in M_{1}^{+}(\mathscr{A})$ is said to be maximal if, for any measure $\nu \in M_{1}^{+}(\mathscr{A})$, the relation $\nu \succ \mu$ implies that $\nu=\mu$.

We need the following properties of Choquet's partial order.

Lemma 2. Let $\mathscr{A}$ be a convex $\mu$-compact set. ${ }^{3}$

(A) If $\mu$ and $\nu$ are measures in $M_{1}^{+}(\mathscr{A})$ such that $\mu \succ \nu$, then

$$
b(\mu)=b(\nu) \quad \text { and } \quad \int_{\mathscr{A}} f(y) \mu(d y) \geq \int_{\mathscr{A}} f(y) \nu(d y) \quad \forall f \in \hat{P}(\mathscr{A}) .
$$

(B) Let $\left\{\mu_{n}\right\}$ and $\left\{\nu_{n}\right\}$ be sequences of measures in $M_{1}^{+}(\mathscr{A})$ converging to some measures $\mu$ and $\nu$, respectively, in $M_{1}^{+}(\mathscr{A})$, satisfying the condition $\mu_{n} \succ \nu_{n}$ for all $n$. Then $\mu \succ \nu$.

(C) If extr $\mathscr{A}=\overline{\text { extr } \mathscr{A}}$, then the set of maximal measures in $M_{1}^{+}(\mathscr{A})$ coincides with the set $M_{1}^{+}(\operatorname{extr} \mathscr{A})$. If, in addition, $\mathscr{A}=\overline{\mathrm{co}}(\operatorname{extr} \mathscr{A})$, Then for each measure $\mu \in M_{1}^{+}(\mathscr{A})$ there exists a maximal measure $\hat{\mu} \in M_{1}^{+}(\mathscr{A})$ such that $\hat{\mu} \succ \mu$.

Proof. Assertions A and B can readily be derived from definitions and the monotone convergence theorem for Lebesgue integral.

The first part of assertion $\mathrm{C}$ can be proved with the use of Theorem 5.2 in [10], Theorem 6.3.9 in [12], and the argument in the proof of Theorem 1.1 in [13]. One can obtain a constructive proof of the second part of assertion $\mathrm{C}$ by using Lemma 1 and Lemma 3 below.

Lemma 3. Let $\mathscr{A}$ be a $\mu$-compact convex set such that extr $\mathscr{A}=\overline{\operatorname{extr} \mathscr{A}}$ and $\mathscr{A}=\overline{\operatorname{co}}(\operatorname{extr} \mathscr{A})$. Then, for each sequence $\left\{\mu_{n}\right\}$ of finitely supported measures in $M_{1}^{+}(\mathscr{A})$ converging to some measure $\mu_{0} \in M_{1}^{+}(\mathscr{A})$, there exists a subsequence $\left\{\mu_{n_{k}}\right\}$ and a sequence $\left\{\hat{\mu}_{k}\right\}$ of measures in $M_{1}^{+}$(extr $\left.\mathscr{A}\right)$ converging to some measure $\hat{\mu}_{0} \in M_{1}^{+}$(extr $\left.\mathscr{A}\right)$ and satisfying the relation

$$
b\left(\hat{\mu}_{k}\right)=b\left(\mu_{n_{k}}\right) \quad \forall k \quad \text { and } \quad \hat{\mu}_{0} \succ \mu_{0} .
$$

If $\mathscr{A}=\sigma-\operatorname{co}(\operatorname{extr} \mathscr{A})$, then for $\left\{\hat{\mu}_{k}\right\}$ one can take a sequence of atomic measures.

Proof. By Proposition 2, for each $n$, every atom $x_{i}^{n}$ of the measure

$$
\mu_{n}=\sum_{i=1}^{m(n)} \pi_{i}^{n} \delta\left(x_{i}^{n}\right)
$$

is the barycenter of some measure

$$
\mu_{i}^{n} \in M_{1}^{+}(\operatorname{extr} \mathscr{A})
$$

It is easily seen that $\hat{\mu}_{n}=\sum_{i=1}^{m(n)} \pi_{i}^{n} \mu_{i}^{n}$ is a measure in $M_{1}^{+}(\operatorname{extr} \mathscr{A})$ such that $b\left(\hat{\mu}_{n}\right)=b\left(\mu_{n}\right)$ and $\hat{\mu}_{n} \succ$ $\mu_{n}$. Since the mapping (1) is continuous, it follows that the set $\left\{b\left(\mu_{n}\right)\right\}_{n \geq 0}$, which coincides with the closure of the set $b\left(\left\{\hat{\mu}_{n}\right\}_{n>0}\right)$, is compact. The set $\mathscr{A}$ is $\mu$-compact; therefore, the sequence $\left\{\hat{\mu}_{n}\right\}_{n>0}$ is relatively compact in $M_{1}^{+}(\operatorname{extr} \mathscr{A})$ and hence contains a subsequence $\left\{\hat{\mu}_{n_{k}}\right\}$ converging to some measure $\left\{\hat{\mu}_{0}\right\} \in M_{1}^{+}(\operatorname{extr} \mathscr{A})$. Since $\hat{\mu}_{n_{k}} \succ \mu_{n_{k}}$ for all $k$, it follows from assertion B in Lemma 2 that $\hat{\mu}_{0} \succ \mu_{0}$. Using the notation $\hat{\mu}_{k}=\hat{\mu}_{n_{k}}$, we obtain the main assertion of the lemma.

If $\mathscr{A}=\sigma-\operatorname{co}(\operatorname{extr} \mathscr{A})$, then the measures $\mu_{i}^{n}$ in the above argument can be chosen to be atomic.

\footnotetext{
${ }^{3}$ The $\mu$-compactness condition is only used in the proof of the second assertion in item C; it permits one to establish the existence of the measure $\hat{\mu}$ without resorting to the Zorn lemma.
} 
Lemma 3 results in the observation that atomic measures are dense in the set of all maximal measures with a given barycenter.

Lemma 4. Let $\mathscr{A}$ be a $\mu$-compact convex set such that extr $\mathscr{A}=\overline{\operatorname{extr} \mathscr{A}}$ and $\mathscr{A}=\sigma$-co(extr $\mathscr{A})$. Then each measure $\mu_{0} \in M_{1}^{+}(\operatorname{extr} \mathscr{A})$ is the limit of a sequence $\left\{\mu_{n}\right\}$ of atomic measures in $M_{1}^{+}(\operatorname{extr} \mathscr{A})$ such that $b\left(\mu_{n}\right)=b\left(\mu_{0}\right)$ for all $n$.

Proof. By Lemma 1, each measure $\mu_{0} \in M_{1}^{+}(\operatorname{extr} \mathscr{A})$ is the limit of a sequence $\left\{\mu_{n}\right\}$ of finitely supported measures in $M_{1}^{+}(\mathscr{A})$ such that $b\left(\mu_{n}\right)=b\left(\mu_{0}\right)$ for all $n$. By applying Lemma 3 and by using the fact (which follows from assertion $\mathrm{C}$ in Lemma 2) that the measure $\mu_{0}$ is maximal in $M_{1}^{+}(\mathscr{A})$, we obtain a sequence $\left\{\hat{\mu}_{n}\right\}$ of measures with the desired properties.

\section{ON THE CONVEX CLOSURE}

For every closed lower-bounded function $f$ on a closed set $\mathscr{A}$, the functional

$$
\mu \mapsto \int_{\mathscr{A}} f(x) \mu(d x)
$$

is well defined and lower semicontinuous on the set $M_{1}^{+}(\mathscr{A})$.

Proposition 3. The convex closure of every lower-bounded closed function $f$ on a $\mu$-compact convex set $\mathscr{A}$ is determined by the expression

$$
\overline{\operatorname{co}} f(x)=\inf _{\mu \in M_{x}(\mathscr{A})} \int_{\mathscr{A}} f(y) \mu(d y) \quad \forall x \in \mathscr{A} .
$$

For each $x \in \mathscr{A}$, there exists a measure $\mu_{x}^{f} \in M_{x}(\mathscr{A})$ such that

$$
\overline{\mathrm{co}} f(x)=\int_{\mathscr{A}} f(y) \mu_{x}^{f}(d y) .
$$

If $\operatorname{extr} \mathscr{A}=\overline{\operatorname{extr} \mathscr{A}}, \mathscr{A}=\overline{\mathrm{co}}(\operatorname{extr} \mathscr{A})$, and $f$ is a concave lower bounded function such that $-f \in \hat{P}(\mathscr{A})$, then for $\mu_{x}^{f}$ one can take a measure supported in extr $\mathscr{A}$.

Remark 1. In general, the infimum over all measures in $M_{x}(\mathscr{A})$ in (3) cannot be replaced by the infimum over all atomic measures in $M_{x}(\mathscr{A})$. (In other words, the integral cannot be replaced by a finite or countable sum.) There exist functions $f$ for which these infima are distinct (see the examples in Remarks 1 and 2 in [14]).

Proof. By assertion A in Lemma 5 below, the function

$$
\check{f}_{\mathscr{A}}=\inf _{\mu \in M_{x}(\mathscr{A})} \int_{\mathscr{A}} f(y) \mu(d y)
$$

is convex and closed on $\mathscr{A}$. Therefore, $\check{f}_{\mathscr{A}}(x) \leq \overline{\mathrm{co}} f(x)$ for all $x \in \mathscr{A}$. Since $\overline{\mathrm{co}} f$ is a convex closed function majorized by $f$, it follows from Jensen's inequality that

$$
\overline{\operatorname{co}} f(x) \leq \inf _{\mu \in M_{x}(\mathscr{A})} \int_{\mathscr{A}} \overline{\operatorname{co}} f(y) \mu(d y) \leq \inf _{\mu \in M_{x}(\mathscr{A})} \int_{\mathscr{A}} f(y) \mu(d y)=\check{f}_{\mathscr{A}}(x)
$$

for all $x \in \mathscr{A}$. Therefore, $\check{f}_{\mathscr{A}}=\overline{\mathrm{co}} f$.

The existence of the measure $\mu_{x}^{f}$ follows from assertion A in Lemma 5.

Let $f$ be a concave lower-bounded function such that $-f \in \hat{P}(\mathscr{A})$, and let $\mu_{x}^{f}$ be the measure whose existence was established above for each $x \in \mathscr{A}$. By assertion $\mathrm{C}$ in Lemma 2, there exists a measure $\hat{\mu}_{x}^{f} \in M_{1}^{+}(\operatorname{extr} \mathscr{A})$ that is maximal in $M_{1}^{+}(\mathscr{A})$ and satisfies $\hat{\mu}_{x}^{f} \succ \mu_{x}^{f}$. By assertion A in Lemma 2, $b\left(\hat{\mu}_{x}^{f}\right)=x$, and the infimum in the expression for $\overline{\mathrm{co}} f(x)$ is attained on $\hat{\mu}_{x}^{f}$. 
Lemma 5. Let $f$ be a closed lower-bounded function on the set $\mathscr{A}$.

(A) If $\mathscr{A}$ is $\mu$-compact, then the function

$$
\check{f}_{\mathscr{A}}(x)=\inf _{\mu \in M_{x}(\mathscr{A})} \int_{\mathscr{A}} f(y) \mu(d y)
$$

is convex and closed on the set $\overline{\mathrm{co}}(\mathscr{A})$; moreover, for each $x \in \overline{\mathrm{co}}(\mathscr{A})$, the infimum in the definition of $\check{f}_{\mathscr{A}}(x)$ is attained on some measure in $M_{x}(\mathscr{A})$.

(B) If the mapping $M_{1}^{+}(\mathscr{A}) \ni \mu \mapsto b(\mu) \in \overline{\mathrm{CO}}(\mathscr{A})$ is open and surjective, then the function

$$
\hat{f}_{\mathscr{A}}(x)=\sup _{\mu \in M_{x}(\mathscr{A})} \int_{\mathscr{A}} f(y) \mu(d y)
$$

is concave and closed on the set $\overline{\mathrm{co}}(\mathscr{A})$.

Proof. (A) The function $\check{f}_{\mathscr{A}}$ is well defined on $\overline{\mathrm{co}}(\mathscr{A})$, since the set $M_{x}(\mathscr{A})$ is nonempty for every element $x \in \overline{\mathrm{co}}(\mathscr{A})$ by Proposition 2 . The convexity of this function readily follows from its definition and the convexity of the set $M_{1}^{+}(\mathscr{A})$. Since the functional (2) is lower semicontinuous and the set $M_{x}(\mathscr{A})$ is compact (the latter follows from the $\mu$-compactness of $\mathscr{A}$ ), it follows that, for each $x \in \overline{\mathrm{co}}(\mathscr{A})$, the infimum in the definition of $\check{f}_{\mathscr{A}}(x)$ is attained at some measure in $M_{x}(\mathscr{A})$.

Suppose that the function $\check{f}_{\mathscr{A}}$ is not closed. Then there exists a sequence $\left\{x_{n}\right\} \subset \overline{\operatorname{co}}(\mathscr{A})$ that converges to $x_{0} \in \overline{\mathrm{co}}(\mathscr{A})$ and satisfies

$$
\lim _{n \rightarrow+\infty} \check{f}\left(x_{n}\right)<\check{f}\left(x_{0}\right) .
$$

By the preceding observation, for each $n=1,2, \ldots$, there exists a measure $\mu_{n} \in M_{x_{n}}(\mathscr{A})$ such that

$$
\check{f}_{\mathscr{A}}\left(x_{n}\right)=\int_{\mathscr{A}} f(y) \mu_{n}(d y) \text {. }
$$

It follows from the $\mu$-compactness of $\mathscr{A}$ and the compactness of the sequence $\left\{x_{n}\right\}_{n \geq 0}$ that the sequence $\left\{\mu_{n}\right\}_{n>0}$ of measures is relatively compact. Consequently, it has a subsequence $\left\{\mu_{n_{k}}\right\}$ converging to some measure $\mu_{0}$. Since the mapping (1) is continuous, it follows that $\mu_{0} \in M_{x_{0}}(\mathscr{A})$. The functional (2) is lower semicontinuous, and so

$$
\check{f}_{\mathscr{A}}\left(x_{0}\right) \leq \int_{\mathscr{A}} f(y) \mu_{0}(d y) \leq \liminf _{k \rightarrow+\infty} \int_{\mathscr{A}} f(y) \mu_{n_{k}}(d y)=\lim _{k \rightarrow+\infty} \check{f}_{\mathscr{A}}\left(x_{n_{k}}\right),
$$

which contradicts (4).

(B) The function $\hat{f}_{\mathscr{A}}$ is well defined on $\overline{\mathrm{co}}(\mathscr{A})$, since, in view of the surjectivity of the barycenter mapping, the set $M_{x}(\mathscr{A})$ is nonempty for each $x \in \overline{\mathrm{co}}(\mathscr{A})$. The concavity of this function is a straightforward consequence of the definition of $\hat{f}_{\mathscr{A}}$ and the convexity of the set $M_{1}^{+}(\mathscr{A})$. Suppose that the function $\hat{f}_{\mathscr{A}}$ is not closed. Then there exists a sequence $\left\{x_{n}\right\} \subset \overline{\mathrm{CO}}(\mathscr{A})$ that converges to $x_{0} \in \overline{\mathrm{CO}}(\mathscr{A})$ and satisfies

$$
\lim _{n \rightarrow+\infty} \hat{f}_{\mathscr{A}}\left(x_{n}\right)<\hat{f}_{\mathscr{A}}\left(x_{0}\right) .
$$

For an arbitrary $\varepsilon>0$, let $\mu_{0}^{\varepsilon} \in M_{x_{0}}(\mathscr{A})$ be a measure such that

$$
\hat{f}_{\mathscr{A}}\left(x_{0}\right)<\int_{\mathscr{A}} f(y) \mu_{0}^{\varepsilon}(d y)+\varepsilon
$$

Since the barycentric mapping is open, it follows that there exists a subsequence $\left\{x_{n_{k}}\right\}$ of the sequence $\left\{x_{n}\right\}$ and a sequence $\left\{\mu_{k}\right\}$ of measures in $M_{1}^{+}(\mathscr{A})$ converging to $\mu_{0}^{\varepsilon}$ such that $b\left(\mu_{k}\right)=x_{n_{k}}$ for each $k$. Since the functional (2) is lower semicontinuous, we have

$$
\hat{f}_{\mathscr{A}}\left(x_{0}\right) \leq \int_{\mathscr{A}} f(y) \mu_{0}^{\varepsilon}(d y)+\varepsilon \leq \liminf _{k \rightarrow+\infty} \int_{\mathscr{A}} f(y) \mu_{k}(d y)+\varepsilon \leq \lim _{k \rightarrow+\infty} \hat{f}_{\mathscr{A}}\left(x_{n_{k}}\right)+\varepsilon
$$

which contradicts (5) in view of the fact that $\varepsilon$ is arbitrary. 
Proposition 3 and Lemma 1 imply the following assertion, which generalizes Corollary I.3.6 in [3] to the case of $\mu$-compact convex sets.

Corollary 1. If $\mathscr{A}$ is a $\mu$-compact convex set, then the convex closure of every function $f \in C(\mathscr{A})$ is determined by the expression

$$
\overline{\mathrm{co}} f(x)=\inf _{n \in \mathbb{N}}\left\{\sum_{i=1}^{n} \pi_{i} f\left(x_{i}\right) \mid \sum_{i=1}^{n} \pi_{i} x_{i}=x,\left\{x_{i}\right\} \subset \mathscr{A},\left\{\pi_{i}\right\} \in \mathfrak{P}_{n}\right\} \quad \forall x \in \mathscr{A} .
$$

This expression means that $\overline{\mathrm{co}} f=\mathrm{co} f$ for any function $f \in C(\mathscr{A})$.

\section{STRONG CE-PROPERTY FOR CONVEX $\mu$-COMPACT SETS}

The CE-property of a convex compact set $\mathscr{A}$ is as follows: the convex closure of every concave function in $C(\mathscr{A})$ belongs to $C(\mathscr{A})[5,6]$. Using this terminology, we introduce the following definition.

Definition 2. A convex topological space $\mathscr{A}$ is said to have the strong CE-property if the convex closure of every function in $C(\mathscr{A})$ belongs to $C(\mathscr{A})$.

It was shown in [5] that the CE-property of a convex compact set $\mathscr{A}$ implies that the set extr $\mathscr{A}$ is closed. Moreover, the CE-property is equivalent to the assertion that the mapping

$$
M_{1}^{+}(\operatorname{extr} \mathscr{A}) \ni \mu \mapsto b(\mu) \in \mathscr{A}
$$

is open. (This mapping is surjective by Choquet's theorem.)

Theorem 1. Let $\mathscr{A}$ be a convex $\mu$-compact set. The following properties are equivalent:

(i) The mapping $\mathscr{A} \times \mathscr{A} \times[0,1] \ni(x, y, \lambda) \mapsto \lambda x+(1-\lambda) y \in \mathscr{A}$ is open.

(ii) The barycentric mapping $M_{1}^{+}(\mathscr{A}) \ni \mu \mapsto b(\mu) \in \mathscr{A}$ is open.

(iii) The set $\mathscr{A}$ has the strong CE-property.

Properties (i)-(iii) imply that the set extr $\mathscr{A}$ is closed and, given $\mathscr{A}=\overline{\mathrm{co}}($ extr $\mathscr{A})$, the mapping

$$
M_{1}^{+}(\operatorname{extr} \mathscr{A}) \ni \mu \mapsto b(\mu) \in \mathscr{A}
$$

is open. ${ }^{4}$

Proof. (i) $\Rightarrow$ (ii) Let us show that property (i) implies that the mapping

$$
\Psi_{n}: \mathscr{A}^{\times n} \times \mathfrak{P}_{n} \ni\left(\left\{x_{i}\right\}_{i=1}^{n},\left\{\pi_{i}\right\}_{i=1}^{n}\right) \mapsto \sum_{i=1}^{n} \pi_{i} x_{i} \in \mathscr{A}
$$

is open. Here

$$
\mathscr{A}^{\times n}=\underbrace{\mathscr{A} \times \cdots \times \mathscr{A}}_{n}
$$

for each $n$. Property (i) means that the mapping $\Psi_{2}$ is open. Suppose that the mapping $\Psi_{n-1}$ is open. In view of symmetry, it suffices to prove that $\Psi_{n}$ is open on the open set

$$
\mathfrak{M}_{0}=\mathscr{A}^{\times n} \times\left(\mathfrak{P}_{n} \backslash\{(\underbrace{0,0, \ldots, 0}_{n-1}, 1)\}\right) .
$$

\footnotetext{
${ }^{4}$ See the remark following the proof of the theorem.

MATHEMATICAL NOTES Vol.82 No.3 2007
} 
One can readily see that the mapping $\Pi_{n}$ given by

$$
\mathfrak{P}_{n} \backslash\{(\underbrace{0,0, \ldots, 0}_{n-1}, 1)\} \ni\left\{\pi_{i}\right\}_{i=1}^{n} \mapsto\left(\left\{\frac{\pi_{i}}{1-\pi_{n}}\right\}_{i=1}^{n-1},\left\{1-\pi_{n}, \pi_{n}\right\}\right) \in \mathfrak{P}_{n-1} \times \mathfrak{P}_{2}
$$

is open.

The fact that the mapping $\Psi_{n}$ is open on $\mathfrak{M}_{0}$ follows from the representation of $\Psi_{n}$ as the product of the mapping $\operatorname{Id}_{\mathscr{A} \times n} \times \Pi_{n}$, which is open on $\mathfrak{M}_{0}$, the mapping

$$
\left(\left\{x_{i}\right\}_{i=1}^{n},\left\{\pi_{i}\right\}_{i=1}^{n-1},\{v, 1-v\}\right) \mapsto\left(\sum_{i=1}^{n-1} \pi_{i} x_{i}, x_{n},\{v, 1-v\}\right)
$$

from $\mathscr{A}^{\times n} \times \mathfrak{P}_{n-1} \times \mathfrak{P}_{2}$ to $\mathscr{A}^{\times 2} \times \mathfrak{P}_{2}$, which is open by the inductive hypothesis, and the mapping $\Psi_{2}$, which is open by property (i).

Let $U$ be an arbitrary open subset of $M_{1}^{+}(\mathscr{A})$. Suppose that the set $b(U)$ is not open. Then, for some $x_{0} \in b(U)$, there exists a sequence $\left\{x_{n}\right\} \subset \mathscr{A} \backslash b(U)$ converging to $x_{0}$.

Let $\mu_{0}$ be a measure in $U$ such that $b\left(\mu_{0}\right)=x_{0}$. By Lemma 1 , we can assume that $\mu_{0}$ is finitely supported; i.e.,

$$
\mu_{0}=\sum_{i=1}^{m} \pi_{i}^{0} \delta\left(x_{i}^{0}\right) \quad \text { for some } \quad\left\{x_{i}^{0}\right\}_{i=1}^{m} \in \mathscr{A}^{m}, \quad\left\{\pi_{i}^{0}\right\}_{i=1}^{m} \in \mathfrak{P}_{m}, \quad m \in \mathbb{N} .
$$

We have already established that the mapping $\Psi_{m}$ is open. Therefore, there exists a subsequence $\left\{x_{n_{k}}\right\}$ of the sequence $\left\{x_{n}\right\}$ and a sequence $\left\{\mu_{k}=\sum_{i=1}^{m} \pi_{i}^{k} \delta\left(x_{i}^{k}\right)\right\}$ of measures converging to $\mu_{0}$ such that

$$
b\left(\mu_{k}\right)=\sum_{i=1}^{m} \pi_{i}^{k} x_{i}^{k}=x_{n_{k}}
$$

for all $k$. Since $U$ is open, it follows that $\mu_{k} \in U$ for all sufficiently large $k$, which contradicts the definition of the sequence $\left\{x_{n}\right\}$.

(ii) $\Rightarrow$ (iii) By Proposition 3, the upper semicontinuity of the function $\overline{\mathrm{co}} f$ under condition (ii) follows from assertion B in Lemma 5, applied to the function $-f$.

(iii) $\Rightarrow$ (i) Let $U_{r}(x)$ be a ball neighborhood of radius $r$ of a point $x \in \mathscr{A}$. One can readily see that property (i) is equivalent to the following: for any $x, y \in \mathscr{A}$, any $\lambda \in[0,1]$, and any sequence $\left\{z_{n}\right\} \subset \mathscr{A}$ converging to $\lambda x+(1-\lambda) y$, there exist points $x_{m} \in U_{1 / m}(x)$ and $y_{m} \in U_{1 / m}(y), m=1,2,3 \ldots$, such that

$$
\lambda_{m} x_{m}+\left(1-\lambda_{m}\right) y_{m} \in\left\{z_{n}\right\}_{n>m} \quad \text { for some } \quad \lambda_{m} \in[0,1] .
$$

Therefore, if property (i) is violated, then there necessarily exist $x_{0}, y_{0} \in \mathscr{A}, \lambda_{0} \in[0,1]$, a sequence $\left\{z_{n}\right\}$ converging to $\lambda_{0} x_{0}+\left(1-\lambda_{0}\right) y_{0}$, and a number $m_{0}$ such that

$$
\operatorname{co}\left(U_{1 / m_{0}}\left(x_{0}\right) \cup U_{1 / m_{0}}\left(y_{0}\right)\right) \cap\left\{z_{n}\right\}_{n>m_{0}}=\varnothing .
$$

Let $f$ be a continuous function on $\mathscr{A}$ with range $[0,1]$ such that

$$
f\left(x_{0}\right)=f\left(y_{0}\right)=0 \quad \text { and } \quad f(x)=1 \quad \forall x \in \mathscr{A} \backslash\left(U_{1 / 2 m_{0}}\left(x_{0}\right) \cup U_{1 / 2 m_{0}}\left(y_{0}\right)\right) .
$$

Assume that the function $\overline{\mathrm{co}} f$, which coincides with the function co $f$ by Corollary 1 , is continuous. Then

$$
\lim _{n \rightarrow+\infty} \operatorname{co} f\left(z_{n}\right)=\operatorname{co} f\left(\lambda_{0} x_{0}+\left(1-\lambda_{0}\right) y_{0}\right)=0 .
$$

For each $n$, every finitely supported measure in $M_{z_{n}}(\mathscr{A})$ can be represented in the form

$$
\sum_{i} \pi_{i}^{n} \delta\left(x_{i}^{n}\right)+\sum_{j} v_{j}^{n} \delta\left(y_{j}^{n}\right)+\sum_{k} \eta_{k}^{n} \delta\left(r_{k}^{n}\right)
$$


where $\left\{x_{i}^{n}\right\} \subset U_{1 / 2 m_{0}}\left(x_{0}\right),\left\{y_{j}^{n}\right\} \subset U_{1 / 2 m_{0}}\left(y_{0}\right),\left\{r_{k}^{n}\right\} \subset \mathscr{A} \backslash\left(U_{1 / 2 m_{0}}\left(x_{0}\right) \cup U_{1 / 2 m_{0}}\left(y_{0}\right)\right)$, and $\left\{\pi_{i}^{n}\right\}$, $\left\{v_{j}^{n}\right\}$, and $\left\{\eta_{k}^{n}\right\}$ are sets of positive numbers such that

$$
\sum_{i} \pi_{i}^{n}+\sum_{j} v_{j}^{n}+\sum_{k} \eta_{k}^{n}=1 \quad \text { and } \quad \sum_{i} \pi_{i}^{n} x_{i}^{n}+\sum_{j} v_{j}^{n} y_{j}^{n}+\sum_{k} \eta_{k}^{n} r_{k}^{n}=z_{n} .
$$

It follows from (7) that for each $n$ there exists a measure of the form (8) such that

$$
\lim _{n \rightarrow+\infty}\left(\sum_{i} \pi_{i}^{n} f\left(x_{i}^{n}\right)+\sum_{j} v_{j}^{n} f\left(y_{j}^{n}\right)+\sum_{k} \eta_{k}^{n} f\left(r_{k}^{n}\right)\right)=0 .
$$

By the definition of $f$, this is possible only if

$$
\lim _{n \rightarrow+\infty} \bar{\eta}_{n}=0, \quad \text { where } \quad \bar{\eta}_{n}=\sum_{k} \eta_{k}^{n} .
$$

Let

$$
\bar{\pi}_{n}=\sum_{i} \pi_{i}^{n}, \bar{v}_{n}=\sum_{j} v_{j}^{n}, \quad \text { and } \quad \bar{r}_{n}=\bar{\eta}_{n}^{-1} \sum_{k} \eta_{k}^{n} r_{k}^{n}
$$

Note that

$$
\bar{x}_{n}=\bar{\pi}_{n}^{-1} \sum_{i} \pi_{i}^{n} x_{i}^{n} \in U_{1 / 2 m_{0}}\left(x_{0}\right) \quad \text { and } \quad \bar{y}_{n}=\bar{v}_{n}^{-1} \sum_{j} v_{j}^{n} y_{j}^{n} \in U_{1 / 2 m_{0}}\left(y_{0}\right),
$$

since the neighborhoods $U_{1 / 2 m_{0}}\left(x_{0}\right)$ and $U_{1 / 2 m_{0}}\left(y_{0}\right)$ are convex.

Without loss of generality, we can assume that $\bar{\pi}_{n} \geq \bar{v}_{n}$ for each $n$. Set

$$
\bar{x}_{n}^{\prime}=\left(\bar{\pi}_{n}+\bar{\eta}_{n}\right)^{-1}\left(\bar{\pi}_{n} \bar{x}_{n}+\bar{\eta}_{n} \bar{r}_{n}\right) \quad \text { and } \quad \bar{\pi}_{n}^{\prime}=\bar{\pi}_{n}+\bar{\eta}_{n} .
$$

Then

$$
z_{n}=\bar{\pi}_{n}^{\prime} \bar{x}_{n}^{\prime}+\bar{v}_{n} \bar{y}_{n} \quad \forall n .
$$

Since the sequence $\left\{\bar{\eta}_{n}\right\}$ tends to zero and the set $\mathscr{A}$ is bounded, it follows that $\bar{x}_{n}^{\prime} \in U_{1 / m_{0}}\left(x_{0}\right)$ for all sufficiently large $n$, which, in view of $\bar{y}_{n} \in U_{1 / 2 m_{0}}\left(y_{0}\right)$ and (9), contradicts (6).

We have thereby proved that properties (i)-(iii) are equivalent.

Property (i) readily implies that the set extr $\mathscr{A}$ is closed.

To prove that properties (i)-(iii) imply that the mapping

$$
M_{1}^{+}(\operatorname{extr} \mathscr{A}) \ni \mu \mapsto b(\mu) \in \mathscr{A}
$$

is open, it suffices to show that, for each measure $\mu_{0} \in M_{1}^{+}(\operatorname{extr} \mathscr{A})$ and each sequence $\left\{x_{n}\right\} \subset \mathscr{A}$ converging to $b\left(\mu_{0}\right)=x_{0}$, there exists a subsequence $\left\{x_{n_{k}}\right\}$ and a sequence $\left\{\mu_{k}\right\}$ of measures in $M_{1}^{+}($extr $\mathscr{A})$ converging to $\mu_{0}$ such that $b\left(\mu_{k}\right)=x_{n_{k}}$ for all $k$. Property (ii) guarantees that, for given measure $\mu_{0}$ and sequence $\left\{x_{n}\right\}$, there exists a subsequence $\left\{x_{n_{k}}\right\}$ and a sequence $\left\{\mu_{k}\right\}$ of measures in $M_{1}^{+}(\mathscr{A})$ converging to $\mu_{0}$ such that $b\left(\mu_{k}\right)=x_{n_{k}}$ for all $k$. By Lemma 1 , we can assume that the sequence $\left\{\mu_{k}\right\}$ consists of finitely supported measures. By Lemma 3 applied to the set $\overline{c o}($ extr $\mathscr{A})$, there exists a subsequence $\left\{x_{n_{k_{m}}}\right\}$ of the sequence $\left\{x_{n_{k}}\right\}$ and a sequence $\left\{\hat{\mu}_{m}\right\}$ of measures in $M_{1}^{+}(\operatorname{extr} \mathscr{A})$ converging to some measure $\hat{\mu}_{0} \in M_{1}^{+}(\operatorname{extr} \mathscr{A})$ such that

$$
b\left(\hat{\mu}_{m}\right)=b\left(\mu_{k_{m}}\right)=x_{n_{k_{m}}} \quad \forall m \quad \text { and } \quad \hat{\mu}_{0} \succ \mu_{0} .
$$

Since $\mu_{0}$ is maximal in $M_{1}^{+}(\mathscr{A})$ (which is guaranteed by Lemma 2 ), it follows that $\hat{\mu}_{0}=\mu_{0}$. Thus, the subsequence $\left\{x_{n_{k_{m}}}\right\}$ and the sequence $\left\{\hat{\mu}_{m}\right\}$ of measures have the desired properties. 
Using Theorem 1, one can prove that the class of convex compact sets in $\mathbb{R}^{n}$ with the strong CEproperty is sufficiently wide and includes the class of $\mathrm{P}$-sets in $\mathbb{R}^{n}$. (The definition can be found in $[2,8]$.) Indeed, by using Theorems 1.8.1 and 1.8.2 in [2], one can readily prove that the mapping

$$
\mathscr{A} \times \mathscr{A} \ni(x, y) \mapsto \lambda x+(1-\lambda) y \in \mathscr{A}
$$

is open for each fixed $\lambda \in[0,1]$ for any $\mathrm{P}$-set $\mathscr{A}$ in $\mathbb{R}^{n}$, which guarantees that property (i) holds for this set. Note that the class of $\mathrm{P}$-sets contains all convex polyhedra and all strictly convex compact sets in $\mathbb{R}^{n}$; furthermore, every convex compact set in $\mathbb{R}^{2}$ is a $\mathrm{P}$-set [2]. However, Example 1.8.1 in [2] shows that, already in $\mathbb{R}^{3}$, not every convex compact set with the strong CE-property is a $\mathrm{P}$-set.

Note also that there exist convex compact sets in $\mathbb{R}^{n}, n \geq 3$, without the strong CE-property. By Theorem 1, any compact set with nonclosed set of extreme points can serve as an example. Protasov (oral communication) proved that property (i) for convex compact sets in $\mathbb{R}^{3}$ is equivalent to the closedness of the set of extreme points and constructed an example of a convex compact set in $\mathbb{R}^{4}$ that has a closed set of extreme points, satisfies the CE-property, but fails to satisfy property (i). Thus, the difference between the CE-property and the strong CE-property of convex compact sets in $\mathbb{R}^{n}$ reveals itself for $n \geq 4$.

It was shown in [5] that the image of any compact set with the CE-property under an open continuous affine mapping also has the CE-property. The counterpart of this assertion for the strong CE-property is established in the corollary given below (with condition (a)), which can readily be proved with the use of the fact that properties (i) and (iii) in Theorem 1 are equivalent.

Corollary 2. Let $\mathscr{A}$ be a $\mu$-compact convex set with the strong CE-property, and let $\Phi$ be a continuous affine mapping of $\mathscr{A}$ onto a $\mu$-compact set $\mathscr{B}$. The set $\mathscr{B}$ has the strong CE-property, provided that one of the following conditions is satisfied:

(a) $\Phi$ is an open mapping.

(b) $\mathscr{B} \subset \mathscr{A}$, and $\Phi^{2}=\Phi$.

By using Corollary 2 with condition (b) and Proposition 4 in Sec. 6, one can establish the strong $\mathrm{CE}$-property of the $\mu$-compact set $\mathfrak{P}_{+\infty}$ of all probability distributions with countably many outcomes. In this case, for $\Phi$ one should take the mapping that takes each density operator to the sequence of its diagonal elements in some fixed basis.

\section{ON CONVEX AND CONCAVE EXTENSIONS}

Let $\mathscr{A}$ be a $\mu$-compact convex set such that extr $\mathscr{A}=\overline{\operatorname{extr} \mathscr{A}}$ and $\mathscr{A}=\overline{\operatorname{co}}(\operatorname{extr} \mathscr{A})$, and let $f$ be a closed lower-bounded function on extr $\mathscr{A}$. By Proposition 2, the set $M_{x}(\operatorname{extr} \mathscr{A})$ of all measures in $M_{1}^{+}(\operatorname{extr} \mathscr{A})$ with barycenter $x$ is nonempty for each $x \in \mathscr{A}$. Consequently, the functions

$$
f_{*}(x)=\inf _{\mu \in M_{x}(\operatorname{extr} \mathscr{A})} \int_{\operatorname{extr} \mathscr{A}} f(y) \mu(d y), \quad f^{*}(x)=\sup _{\mu \in M_{x}(\operatorname{extr} \mathscr{A})} \int_{\operatorname{extr} \mathscr{A}} f(y) \mu(d y)
$$

are well defined on $\mathscr{A}$.

Since the set $M_{1}^{+}(\operatorname{extr} \mathscr{A})$ is convex, it follows that the first of these functions is convex and the second is concave on $\mathscr{A}$. The functions $f_{*}$ and $f^{*}$ coincide with $f$ on extr $\mathscr{A}$. Hence they can be viewed as the convex and concave extensions, respectively, of $f$ to $\mathscr{A}$. The properties of these extensions are described in the following theorem.

Theorem 2. Let $\mathscr{A}$ be a convex $\mu$-compact set such that extr $\mathscr{A}=\overline{\operatorname{extr} \mathscr{A}}$ and $\mathscr{A}=\overline{\mathrm{co}}(\operatorname{extr} \mathscr{A})$, and let $f$ be a closed lower-bounded function on extr $\mathscr{A}$.

(A) The function $f_{*}$ is the maximal convex closed extension of $f$ to $\mathscr{A} .{ }^{5}$ For each $x \in \mathscr{A}$, there exists a measure $\hat{\mu}_{x}^{f} \in M_{x}(\operatorname{extr} \mathscr{A})$ such that

$$
f_{*}(x)=\int_{\operatorname{extr} \mathscr{A}} f(y) \hat{\mu}_{x}^{f}(d y)
$$

\footnotetext{
${ }^{5}$ In [3], this function is called the lower envelope of $f$.
} 
(B) If the mapping $M_{1}^{+}(\operatorname{extr} \mathscr{A}) \ni \mu \mapsto b(\mu) \in \mathscr{A}$ is open, then the function $f^{*}$ is closed. If, in addition, $\mathscr{A}=\sigma-\operatorname{co}(\operatorname{extr} \mathscr{A})$, then the function $f^{*}$ is the minimal concave lower-bounded extension of $f$ to $\mathscr{A}$, and

$$
f^{*}(x)=\sup \left\{\sum_{i=1}^{+\infty} \pi_{i} f\left(x_{i}\right) \mid \sum_{i=1}^{+\infty} \pi_{i} x_{i}=x,\left\{x_{i}\right\} \subset \operatorname{extr} \mathscr{A},\left\{\pi_{i}\right\} \in \mathfrak{P}_{\infty}\right\} \quad \forall x \in \mathscr{A} .
$$

Remark 2. By Theorem 1, all main assumptions of Theorem 2 are satisfied for a convex $\mu$-compact set $\mathscr{A}$ that has the strong $\mathrm{CE}$-property and satisfies $\mathscr{A}=\overline{\mathrm{co}}($ extr $\mathscr{A})$.

Analogs of the assertions in Theorem 2 on the existence of the measure $\hat{\mu}_{x}^{f}$ and on the representation of the function $f^{*}$ in the case $\mathscr{A}=\sigma-\operatorname{co}(\operatorname{extr} \mathscr{A})$ do not hold for the functions $f^{*}$ and $f_{*}$, respectively (see the examples in Remark 2 in [14]). The lower boundedness condition in assertion B is important. Indeed, the function

$$
g(x)= \begin{cases}f^{*}(x), & x \in \operatorname{co}(\operatorname{extr} \mathscr{A}) \\ -\infty, & x \in \mathscr{A} \backslash \operatorname{co}(\operatorname{extr} \mathscr{A})\end{cases}
$$

is a concave extension of $f$ to $\mathscr{A}$ and is majorized by $f^{*}$.

Proof. Lemma 5 (for the set extr $\mathscr{A}$ ), together with the $\mu$-compactness of the set extr $\mathscr{A} \subset \mathscr{A}$ and Proposition 2, implies the closedness of $f_{*}$ and $f^{*}$ and the existence of $\hat{\mu}_{x}^{f}$.

Let $g$ be a convex closed lower-bounded extension of $f$ to $\mathscr{A}$. By Jensen's inequality,

$$
g(x) \leq \inf _{\mu \in M_{x}(\operatorname{extr} \mathscr{A})} \int_{\operatorname{extr} \mathscr{A}} g(y) \mu(d y)=\inf _{\mu \in M_{x}(\operatorname{extr} \mathscr{A})} \int_{\operatorname{extr} \mathscr{A}} f(y) \mu(d y)=f_{*}(x)
$$

for all $x \in \mathscr{A}$. Hence $f_{*}$ is the maximal convex closed extension of $f$ to $\mathscr{A}$.

If $\mathscr{A}=\sigma-\operatorname{co}($ extr $\mathscr{A})$, then it follows from Lemma 4 that atomic measures are dense in $M_{x}($ extr $\mathscr{A})$ for each $x \in \mathscr{A}$. This, together with the lower semicontinuity of the functional (2), readily shows that it suffices to take the supremum in the definition of $f^{*}(x)$ over the set of atomic measures in $M_{x}(\operatorname{extr} \mathscr{A})$.

Let $g$ be a lower bounded concave extension of $f$ to $\mathscr{A}$. By applying Jensen's inequality to this function, we obtain

$$
\begin{aligned}
g(x) & \geq \sup \left\{\sum_{i=1}^{+\infty} \pi_{i} g\left(x_{i}\right) \mid \sum_{i=1}^{+\infty} \pi_{i} x_{i}=x,\left\{x_{i}\right\} \subset \operatorname{extr} \mathscr{A},\left\{\pi_{i}\right\} \in \mathfrak{P}_{\infty}\right\} \\
& =\sup \left\{\sum_{i=1}^{+\infty} \pi_{i} f\left(x_{i}\right) \mid \sum_{i=1}^{+\infty} \pi_{i} x_{i}=x,\left\{x_{i}\right\} \subset \operatorname{extr} \mathscr{A},\left\{\pi_{i}\right\} \in \mathfrak{P}_{\infty}\right\}=f^{*}(x)
\end{aligned}
$$

for all $x \in \mathscr{A}$. Hence $f^{*}$ is the minimal lower-bounded concave extension of $f$ to $\mathscr{A}$.

Theorem 2 implies the following generalization of Proposition 9 in [5] to the case of $\mu$-compact convex sets.

Corollary 3. Suppose that $\mathscr{A}$ is a convex $\mu$-compact set with the strong CE-property such that $\mathscr{A}=\overline{\mathrm{co}}($ extr $\mathscr{A})$.

For each function $f \in C(\operatorname{extr} \mathscr{A})$, the function $f_{*}$ defined by the first formula in (10) is a continuous bounded convex extension of $f$ to $\mathscr{A}$ and coincides with the supremum of all convex closed extensions of $f$ to $\mathscr{A}$. If, in addition, $\mathscr{A}=\sigma-\operatorname{co}(\operatorname{extr} \mathscr{A})$, then

$$
f_{*}(x)=\inf \left\{\sum_{i=1}^{+\infty} \pi_{i} f\left(x_{i}\right) \mid \sum_{i=1}^{+\infty} \pi_{i} x_{i}=x,\left\{x_{i}\right\} \subset \operatorname{extr} \mathscr{A},\left\{\pi_{i}\right\} \in \mathfrak{P}_{\infty}\right\} \quad \forall x \in \mathscr{A},
$$

and $f_{*}$ coincides with the supremum of all convex upper-bounded extensions of $f$ to $\mathscr{A}$. 
For the function $f^{*}$, one can state an assertion dual to the assertion in Corollary 3.

Note that if a set $\mathscr{A}$ satisfying the assumptions of Corollary 3 is not compact, then it is not obvious at all that every function in $C($ extr $\mathscr{A})$ has at least one convex extension in $C(\mathscr{A})$. For compact $\mathscr{A}$, this property is equivalent to the closedness of the set extr $\mathscr{A}$ [5, Corollary 2].

Note the following criterion, to be used in the next section, for the continuity of the convex closure of concave functions.

Corollary 4. Suppose that $\mathscr{A}$ is a convex $\mu$-compact set that has the strong CE-property and satisfies $\mathscr{A}=\overline{\mathrm{co}}($ extr $\mathscr{A})$.

Let $f$ be a concave lower-bounded closed function on $\mathscr{A}$ such that $-f \in \hat{P}(\mathscr{A})$. Then

$$
\{\overline{\operatorname{co}} f \in C(\mathscr{A})\} \Leftrightarrow\left\{\left.f\right|_{\text {extr } \mathscr{A}} \in C(\operatorname{extr} \mathscr{A})\right\} .
$$

Proof. By Proposition 3, in view of the last assertion in Theorem 1,

$$
\overline{\mathrm{co}} f(x)=\inf _{\mu \in M_{x}(\operatorname{extr} \mathscr{A})} \int_{\operatorname{extr} \mathscr{A}} f(y) \mu(d y)=f_{*}(x) \quad \forall x \in \mathscr{A}
$$

for each function $f$ of the form indicated. If $f$ is continuous and bounded on extr $\mathscr{A}$, then, by Corollary 3 , the function $\overline{\mathrm{co}} f=f_{*}$ is continuous and bounded on $\mathscr{A}$. The converse is obvious, since the functions $f$ and $\overline{\mathrm{co}} f$ coincide on extr $\mathscr{A}$ by Proposition 3.

\section{SOME APPLICATIONS}

Let $\mathscr{H}$ be a separable Hilbert space, and let $\mathfrak{T}(\mathscr{H})$ be the separable Banach space of all trace-class operators in $\mathscr{H}$ with the trace norm $\|A\|_{1}=\operatorname{Tr} \sqrt{A^{*} A}$. A density operator (a quantum state) is a positive trace class operator $\rho$ with unit trace $[4,15]$. The set $\mathfrak{S}(\mathscr{H})$ of all density operators is a convex closed subset of $\mathfrak{T}(\mathscr{H})$; it is compact if and only if $\mathscr{H}$ is finite-dimensional. The set extr $\mathfrak{S}(\mathscr{H})$ is closed and consists of pure states (one-dimensional projections). Moreover, $\mathfrak{S}(\mathscr{H})=\sigma-\operatorname{co}(\operatorname{extr} \mathfrak{S}(\mathscr{H})$ ) by the spectral theorem. The pure state equal to the projection onto the subspace spanned by a unit vector $\varphi$ will be denoted by $P_{\varphi}$.

Using the sufficient condition given in Proposition 1, one can prove the $\mu$-compactness of $\mathfrak{S}(\mathscr{H})$. To this end, for $F(\mathfrak{S}(\mathscr{H}))$ one can take a family of functions of the form $\rho \mapsto \operatorname{Tr} H \rho$, where $H$ is an arbitrary unbounded positive operator with discrete spectrum of finite multiplicity. (See [11] for details.) Using Lemma 3 in [16] with $m=2$ and Theorem 1, we arrive at the following assertion.

Proposition 4. The set $\mathfrak{S}(\mathscr{H})$ is a convex $\mu$-compact set with the strong $\mathrm{CE}$-property.

Note that the $\mu$-compactness of $\mathfrak{S}(\mathscr{H})$, together with Lemma 4 , implies a useful result stating that atomic measures are dense in the set of all probability measures on extr $\mathfrak{S}(\mathscr{H})$ with a given barycenter: each measure $\mu_{0}$ in $M_{1}^{+}(\operatorname{extr} \mathfrak{S}(\mathscr{H}))$ is the limit of a sequence $\left\{\mu_{n}\right\}$ of atomic measures in $M_{1}^{+}(\operatorname{extr} \mathfrak{S}(\mathscr{H}))$ such that $b\left(\mu_{n}\right)=b\left(\mu_{0}\right)$ for all $n$.

The von Neumann entropy of a density operator $\rho$ is defined by the expression

$$
H(\rho)=-\sum_{i} \lambda_{i} \log \lambda_{i}
$$

where $\left\{\lambda_{i}\right\}$ is the set of eigenvalues of $\rho$, and is a closed concave function on $\mathfrak{S}(\mathscr{H})$ ranging in $[0,+\infty][17]$.

Consider a composite quantum system whose states are density operators in the tensor product $\mathscr{H} \otimes \mathscr{K}$ of two separable Hilbert spaces $\mathscr{H}$ and $\mathscr{K}$ characterizing separate subsystems. Consider the partial entropy, which is the closed concave function

$$
f_{\mathscr{H}}: \omega \mapsto H\left(\operatorname{Tr}_{\mathscr{K}} \omega\right) \in[0,+\infty]
$$


on $\mathfrak{S}(\mathscr{H} \otimes \mathscr{K})$, where $\operatorname{Tr}_{\mathscr{K}}(\cdot)$ is the partial trace over the space $\mathscr{K}$ [4]. The value of the convex closure of $f_{\mathscr{H}}$ on a state $\omega \in \mathfrak{S}(\mathscr{H} \otimes \mathscr{K})$ is an important characteristic of this state. It is called the entanglement of formation $(\mathrm{EoF})$ and is denoted by $E_{F}(\omega)[18,19]$; i.e.,

$$
E_{F}(\omega)=\overline{\mathrm{co}} f_{\mathscr{H}}(\omega) \quad \forall \omega \in \mathfrak{S}(\mathscr{H} \otimes \mathscr{K}) .
$$

One can readily show that $E_{F}(\omega)=0$ if and only if $\omega$ is a nonentangled state, i.e., lies in the convex closure of the set of pure product states.

In a similar way, one can define the function $f_{\mathscr{K}}: \omega \mapsto H\left(\operatorname{Tr}_{\mathscr{H}} \omega\right)$. Although the functions $f_{\mathscr{H}}$ and $f_{\mathscr{K}}$ are distinct, they coincide on the set extr $\mathfrak{S}(\mathscr{H} \otimes \mathscr{K})[4]$. Hence the definition of the EoF is independent of the choice of the space over which the partial trace is taken; i.e.,

$$
E_{F}=\overline{\operatorname{co}} f_{\mathscr{H}}=\overline{\operatorname{co}} f_{\mathscr{K}} .
$$

The following observations concerning the properties of the EoF can be obtained from these results in view of Proposition 4.

Proposition 5. (A) The EoF is the maximal closed convex function coinciding with the partial entropy on the set of pure states.

(B) Let $\mathscr{L}$ be a subspace of $\mathscr{H} \otimes \mathscr{K}$. The EoF is continuous and bounded on the set

$$
\mathfrak{S}(\mathscr{L})=\{\omega \in \mathfrak{S}(\mathscr{H} \otimes \mathscr{K}) \mid \operatorname{supp} \omega \subseteq \mathscr{L}\}
$$

if and only if the function

$$
\varphi \mapsto f_{\mathscr{H}}\left(P_{\varphi}\right)=H\left(\operatorname{Tr}_{\mathscr{K}} P_{\varphi}\right)
$$

is continuous and bounded on the unit sphere in $\mathscr{L}$.

(C) The EoF is continuous and bounded on $\mathfrak{S}(\mathscr{H} \otimes \mathscr{K})$ if and only if either $\operatorname{dim} \mathscr{H}<+\infty$ or $\operatorname{dim} \mathscr{K}<+\infty$. $^{6}$

Proof. Assertion A follows from Proposition 3 and assertion A of Theorem 2.

Assertion B follows from Corollary 4.

Assertion C follows from B in view of (11).

Assertion A in Proposition 5 is of interest in connection with the fact that each function serving as a candidate for a measure of entanglement should coincide on the set of pure states with the partial entropy $f_{\mathscr{H}}[5]$.

If $\mathscr{H}$ and $\mathscr{K}$ are infinite-dimensional spaces, then the $\mathrm{EoF}$ is not a continuous function on $\mathfrak{S}(\mathscr{H} \otimes$ $\mathscr{K})$, but there exists subspaces $\mathscr{L} \subseteq \mathscr{H} \otimes \mathscr{K}$ such that although the functions $f_{\mathscr{H}}$ and $f_{\mathscr{K}}$ are unbounded on the set $\mathfrak{S}(\mathscr{L})$, the assumption in assertion B in Proposition 5 is satisfied. By this assertion, the EoF is continuous and bounded on the set $\mathfrak{S}(\mathscr{L})$. This result is important in that, by virtue of an observation in [21], the output entropy of any quantum channel can be represented as the restriction of the EoF to the set $\mathfrak{S}(\mathscr{L})$ for some $\mathscr{L} \subseteq \mathscr{H} \otimes \mathscr{K}$. In practice, one can prove that the assumption in assertion B holds for a given subspace $\mathscr{L}$ by using a sufficient condition for the continuity of quantum entropy for noncompact sets of states [22].

From Corollary 3, in view of Proposition 4 and the spectral theorem, we obtain the following assertion, which can be used for constructing continuous convex (or concave) characteristics of quantum states.

Corollary 5. For each function $f \in C(\operatorname{extr} \mathfrak{S}(\mathscr{H}))$, the function

$$
f_{*}(\rho)=\inf \left\{\sum_{i=1}^{+\infty} \pi_{i} f\left(\rho_{i}\right) \mid \sum_{i=1}^{+\infty} \pi_{i} \rho_{i}=\rho,\left\{\rho_{i}\right\} \subset \operatorname{extr} \mathfrak{S}(\mathscr{H}),\left\{\pi_{i}\right\} \in \mathfrak{P}_{\infty}\right\}
$$

is a convex continuous extension of $f$ to the set $\mathfrak{S}(\mathscr{H})$. This function coincides with the supremum of all convex closed extensions of $f$ to $\mathfrak{S}(\mathscr{H})$ and with the supremum of all convex upper-bounded extensions of $f$ to $\mathfrak{S}(\mathscr{H})$.

\footnotetext{
${ }^{6}$ Note that the direct proof of continuity of the EoF for the case in which $\operatorname{dim} \mathscr{H}<+\infty$ and $\operatorname{dim} \mathscr{K}<+\infty$ is not trivial [20]. 
Since the set extr $\mathfrak{S}(\mathscr{H})$ consists of pure states (one-dimensional projections), it follows that the set $C(\operatorname{extr} \mathfrak{S}(\mathscr{H}))$ is isomorphic to the set of continuous bounded functions defined on the unit sphere of the Hilbert space $\mathscr{H}$ and invariant with respect to multiplication of the argument by unimodular complex numbers.

Using Corollary 5 , one can construct a continuous bounded quasi-measure of entanglement, ${ }^{7}$ closely related to the entanglement of formation $E_{F}$. Let $n \in \mathbb{N}$. For an arbitrary pure state $\omega \in \mathfrak{S}(\mathscr{H} \otimes \mathscr{K})$, let

$$
f_{\mathscr{H}}^{n}(\omega)=-\sum_{i=1}^{n} \lambda_{i} \log \lambda_{i}+\left(\sum_{i=1}^{n} \lambda_{i}\right) \log \left(\sum_{i=1}^{n} \lambda_{i}\right),
$$

where $\left\{\lambda_{i}\right\}_{i=1}^{n}$ is the set of $n$ largest eigenvalues (with multiplicities taken into account) of the states $\operatorname{Tr}_{\mathscr{K}} \omega \cong \operatorname{Tr}_{\mathscr{H}} \omega$. By Proposition 4 in [17], the sequence $\left\{f_{\mathscr{H}}^{n}\right\}$ of functions in $C(\operatorname{extr} \mathfrak{S}(\mathscr{H} \otimes \mathscr{K}))$ is increasing and converges pointwise to the partial entropy $f_{\mathscr{H}}$.

Let $E_{F}^{n}$ be the extension $\left(f_{\mathscr{H}}^{n}\right)_{*}$ of the function $f_{\mathscr{H}}^{n}$ to the set $\mathfrak{S}(\mathscr{H} \otimes \mathscr{K})$ defined in Corollary 5 for $f=f_{\mathscr{H}}^{n}$. By this corollary, $E_{F}^{n}$ belongs to $P(\mathfrak{S}(\mathscr{H} \otimes \mathscr{K}))$. By construction, the function $E_{F}^{n}$ has the following properties:

1. $E_{F}^{n}(\omega)=0$ if and only if $\omega$ is a nonentangled state.

2. $E_{F}^{n}(\omega) \leq E_{F}(\omega)$ for each state $\omega$.

3. $E_{F}^{n}(\omega)=E_{F}(\omega)$ for each state $\omega$ such that either $\operatorname{rank} \operatorname{Tr}_{\mathscr{K}} \omega \leq n$ or $\operatorname{rank} \operatorname{Tr}_{\mathscr{H}} \omega \leq n$.

4. $0 \leq E_{F}^{n}(\omega) \leq \log n$ for each state $\omega$.

The sequence $\left\{E_{F}^{n}\right\}$ of functions in $P(\mathfrak{S}(\mathscr{H} \otimes \mathscr{K}))$ is increasing. Consequently, its pointwise limit $E_{F}^{+\infty}$ is a convex closed function on $\mathfrak{S}(\mathscr{H} \otimes \mathscr{K})$ and coincides on the set of pure states with the partial entropy. It is of interest to study the relation between $E_{F}^{+\infty}$ and $E_{F}$. By construction, we have $E_{F}^{+\infty}(\omega) \leq E_{F}(\omega)$ for an arbitrary state $\omega$, where the equality occurs for each pure state $\omega$ and also, by property 3 , for each state $\omega$ such that either $\operatorname{rank} \operatorname{Tr}_{\mathscr{K}} \omega \leq+\infty$ or $\operatorname{rank} \operatorname{Tr}_{\mathscr{H}} \omega \leq+\infty$. Proving that $E_{F}^{+\infty}$ and $E_{F}$ coincide on the entire $\mathfrak{S}(\mathscr{H} \otimes \mathscr{K})$ would mean that $E_{F}$ is a function of the class $\hat{P}(\mathfrak{S}(\mathscr{H} \otimes \mathscr{K})){ }^{8}$

\section{POSSIBLE GENERALIZATIONS}

The conditions that the set $\mathscr{A}$ is separable, metrizable, and $\mu$-compact are substantially used when proving the main results of the paper. Since these conditions are rather restrictive, let us briefly discuss possible generalizations of these results to a wider class of convex sets.

First, note that the main result of the paper, Theorem 1, can be generalized in a straightforward manner to the class of compact convex subsets of an arbitrary locally convex linear topological space. To obtain this generalization, one should use Proposition I.2.3 in [3] instead of Lemma 1 and slightly modify the proof of Theorem 1. However, it is very difficult in this case to weaken the compactness requirements.

Note also that one can prove the equivalence of properties (i) and (iii) in Theorem 1 where the condition of $\mu$-compactness of the set is replaced by the condition that the convex closure and the convex envelope of an arbitrary continuous bounded function on this set coincide.

\footnotetext{
${ }^{7}$ As was noted above, each measure of entanglement coincides on the set of pure states with the partial entropy and hence cannot be continuous in the infinite-dimensional case.

${ }^{8}$ This conjecture has now been proved by the author.
} 


\section{ACKNOWLEDGMENTS}

The author wishes to express gratitude to V. M. Tikhomirov and the participants of his seminar, especially V. Yu. Protasov, as well as to E. S. Polovinkin and M. V. Balashov for interesting discussions. The author is grateful to the referee for useful remarks, which were taken into account in the final version of the paper.

This work was supported by the program "Modern Problems of Theoretical Mathematics" of the Branch of Mathematics of the Russian Academy of Sciences and the Russian Foundation for Basic Research (grant no. 06-01-00164-a).

\section{REFERENCES}

1. A. D. Ioffe and V. M. Tikhomirov, Theory of Extremal Problems (Nauka, Moscow, 1974; North-Holland, Amsterdam-New York, 1979).

2. E. S. Polovinkin and M. V. Balashov, Elements of Convex and Strongly Convex Analysis (Fizmatlit, Moscow, 2004) [in Russian].

3. E. Alfsen, Compact Convex Sets and Boundary Integrals, in Ergebnisse der Mathematik und ihrer Grenzgebiete (Springer-Verlag, New York-Heidelberg, 1971), Vol. 57.

4. A. S. Kholevo, Introduction to Quantum Information Theory (MCCME, Moscow, 2002) [in Russian].

5. A. Lima, "On continuous convex functions and split faces," Proc. London Math. Soc. (3) 25 (3), 27-40 (1972).

6. S. S. Kutateladze, "Choquet boundaries in $K$-spaces," Uspekhi Mat. Nauk 30 (4), 107-146 (1975) [Russian Math. Surveys 30 (4), 115-155(1975)].

7. R. R. Phelps, Lectures on Choquet's Theorem (Van Nostrand Co., Princeton-Toronto-London, 1966; Mir, Moscow, 1968)

8. M. V. Balashov, "On the P-property of convex compact sets," Mat. Zametki 71 (3), 323-333 (2002) [Math. Notes $71(3-4), 295-304(2002)]$.

9. K. Parthasarathy, Probability Measures on Metric Spaces, in Probability and Mathematical Statistics (Academic Press, New York-London, 1967), Vol. 3.

10. G. A. Edgar, "Extremal integral representations," J. Funct. Anal. 23 (2), 145-161 (1976).

11. A. S. Kholevo and M. E. Shirokov, "Continuous ensembles and the capacity of infinite-dimensional quantum channels," Teor. Veroyatnost. i Primenen. 50 (1), 98-114 (2006) [Theory Probab. Appl. 50 (1), 86-98 (2006)]; arXiv: quant-ph/0408176.

12. R. D. Bourgin, Geometric Aspects of Convex Sets with the Radon-Nikodym Property, in Lecture Notes in Math. (Springer-Verlag, Berlin, 1983), Vol. 993.

13. R. D. Bourgin and G. A. Edgar, "Noncompact simplexes in Banach spaces with the Radon-Nikodym property," J. Funct. Anal. 23 (2), 162-176 (1976).

14. M. E. Shirokov, Properties of Probability Measures on the set of Quantum States and Their Applications, arXiv: math-ph/0607019.

15. M. Keyl, "Fundamentals of Quantum Information Theory," Phys. Rep. 369 (5), 431-548 (2002); arXiv: quant-ph/0202122.

16. M. E. Shirokov, "The Holevo capacity of infinite-dimensional channels and the additivity problem," Comm. Math. Phys. 262 (1), 137-159 (2006); arXiv: quant-ph/0408009.

17. G. Lindblad, "Expectation and Entropy Inequalities for Finite Quantum Systems," Comm. Math. Phys. 39 (2), 111-119(1974).

18. C. H. Bennett, D. P. DiVincenzo, J. A. Smolin, and W. K. Wootters, "Mixed-State Entanglement and Quantum Error Correction,” Phys. Rev. A (3) 54 (5), 3824-3851 (1996); arXiv: quant-ph/9604024.

19. M. E. Shirokov, On Entropic Quantities Related to the Classical Capacity of Infinite-Dimensional Quantum Channels, arXiv: quant-ph/0411091.

20. M. A. Nielsen, "Continuity bounds for entangelment," Phys. Rev. A (3) 61 (6) (2000).

21. K. Matsumoto, T. Shimono, and A. Winter, "Remarks on additivity of the Holevo channel capacity and of the entanglement of formation," Comm. Math. Phys. 246 (3), 427-442 (2004).

22. M. E. Shirokov, The Convex Closure of the Output Entropy of Infinite-Dimensional Channels and the Additivity Problem, arXiv: quant-ph/0608090. 